\title{
Computer simulation and experimental investigation of solidification casting process
}

\begin{abstract}
Computer simulation is widely used and conventional in manufacturing as a way to improve the manufactured goods quality as at same time plummeting invention costs, development time and scrap. In this study, three-dimensional model have been developed to simulate the filling and the solidification pattern in an aluminum casting with ñAnyCastingò software. Using this software, solidification of aluminium $11.8 \% \mathrm{Si}$ alloy in sand and metal mould was simulated and the results were compared with the experimental results. The solidification time and temperature was experimentally determined from thermocouple located at diverse distances. A data logger is used in experiment to gain the temperature division contour during the process of solidification of the aluminium $11.8 \% \mathrm{Si}$ alloy. Experiments were set up to legalize the simulation results and it is confirm that the results from the two mould methods be in good agreement and the solidification of cast aluminium alloy using copper mould was much faster compared to the one cast sand mould. From this work it is concluded that simulation using AnyCasting software can be use to initially calculate. The simulation shows result that matches with the experimental data qualitatively and therefore the validation procedure is successful.
\end{abstract}

Keyword: Casting process; Computer simulation; Filling and solidification 